\title{
Pathogenesis of intracranial germ cell tumors reconsidered
}

Keiji Sano, M.D., D.M.Sc.

Department of Neurosurgery, Fuji Brain Institute, University of Tokyo, and Department of Neurosurgery, Teikyo University, Tokyo, Japan

The author studied 153 cases of intracranial germ cell tumors (GCTs) through 1994, 62.7\% of which showed monotypic histological patterns and 37.3\% of which were shown to be mixed tumors. All of these cases, except for six patients who died soon after admission and underwent autopsy, underwent surgery followed by radio- and/or chemotherapy. All patients with choriocarcinoma died within 2 years. Patients with yolk sac tumor (endodermal sinus tumor) and embryonal carcinoma also had poor outcomes. Patients with mature teratoma had 5- and 10-year survival rates of $92.9 \%$ each. Patients with immature teratoma and malignant teratoma had a 5- and 10-year survival rate of $70.7 \%$ each. Patients with germinoma had a 5-year survival rate of $95.4 \%$ and a 10 -year survival rate of $92.7 \%$. These results may bring into question the validity of the germ cell theory, because germinoma, which should be the most undifferentiated according to the theory, was the most benign and choriocarcinoma and yolk sac tumor (endodermal sinus tumor), which should be the most differentiated, were the most malignant according to results obtained during follow-up study.

Therefore, GCTs other than germinoma may not originate from one single type of cell (primordial germ cells). The embryonic cells of various stages of embryogenesis may perhaps be misplaced in the bilaminar embryonic disc at the time of the primitive streak formation, becoming involved in the stream of lateral mesoderm and carried to the future cranial area to become incorrectly enfolded into the brain at the time of the neural tube formation. The authors propound the following law: tumors composed of cells resembling the cells that appear in the earlier stages of embryogenesis (ontogenesis) are more malignant than those composed of cells resembling the cells that appear in the later stages of embryogenesis.

Key Words * embryogenesis * germinoma * germ cell tumor * survival rate

\section{GERM CELL TUMORS: WHAT ARE THEY?}

In 1944, Russell[20] published the results of a study on pineal tumors, providing a seemingly rational explanation for the similarity in histological appearance of two-cell pattern "pinealomas" and "seminomas." She suggested that many pinealomas were in truth teratoid tumors (atypical teratomas). In 1946, Friedman and Moore,[10] having examined 922 tumors of the testis, concluded that seminomas (and dysgerminomas) were probably tumors of primordial germ cells that should be termed 
"germinomas." Friedman,[8,9] in the following years, stressed that the two-cell pattern pinealoma was really the germinoma and that the embryonal carcinoma developed from the germinoma and, in turn, gave rise to the teratoma and the chorioepithelioma (choriocarcinoma). Dixon and Moore[7] stated that germ cells gave rise to germinoma and, alternatively, to embryonal carcinoma from which choriocarcinoma and teratoma derived. Teilum, [27,28] establishing endodermal sinus tumors (yolk sac tumors), summed up the so-called germ cell theory: germ cells give rise to germinomas and, along another line, to tumors comprised of totipotent cells which give rise to embryonal carcinomas and from which endodermal sinus tumors, choriocarcinomas, and teratomas derive (Fig. 1). This theory received the approval of Rubinstein[19] and the World Health Organization Committee of brain tumor classification.[13]

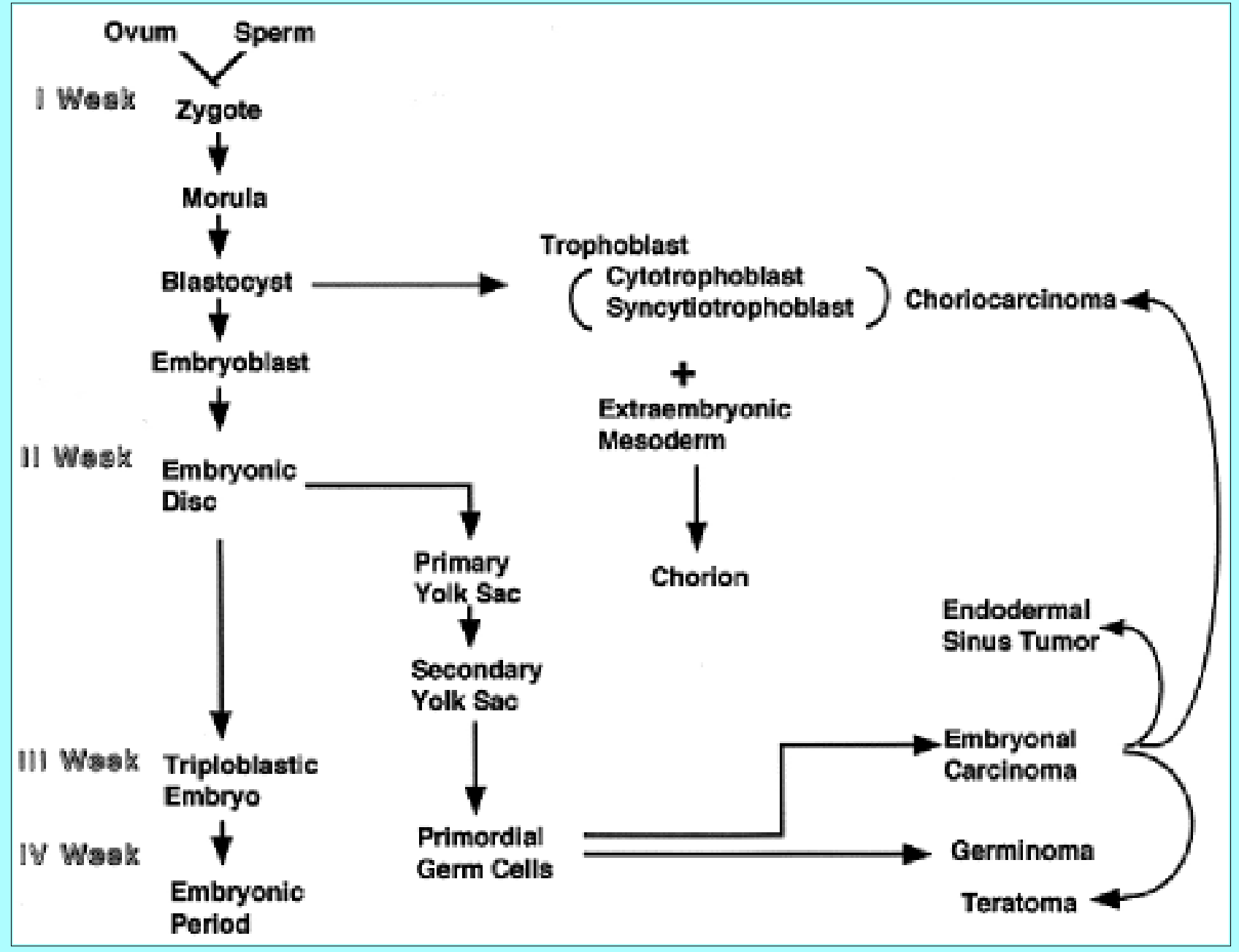

Fig. 1. Diagram depicting germ cell theory, showing the twisted, improbable pathogenetic lines. (Reprinted with permission of Sano K: So-called intracranial germ cell tumours: are they really of germ cell origin? Br J Neurosurg 9:391-401, 1995.)

Although there have been divergent views such as this author's assertion[21,23] that there are two types of intracranial (particularly pineal) two-cell pattern tumors (namely, germinoma and pineocytoma with lymphocytic infiltration) and Zülch's[32] report that these two types of two-cell pattern tumors can be differentiated by Di Girolami impregnation and periodic acid Schiff stain, the trend now is in favor of denying the duality of the two-cell pattern tumors and affirming the germ cell origin of all these tumors.

Therefore, under the term "germ cell tumors" (GCTs), whether gonadal or extragonadal, it is now customary to include germinoma (seminoma or dysgerminoma), mature and immature teratoma, 
malignant teratoma or teratoma with malignant transformation, embryonal carcinoma, yolk sac tumor (endodermal sinus tumor), choriocarcinoma, and so-called mixed GCTs (tumors consisting of two or more of these tumor components).[13] This classification, based on the "germ cell theory," maintains that these tumors originate from the germ cells. Whether the "germ cells" correspond to the primordial germ cells known in embryology $[4,9,10,26]$ or to certain stages of differentiation of the primordial germ cells is not clear. Takei and Pearl[26] adopted Mostofi's[18] concept of totipotent malignant germ cells and proposed a schema of possible oncological phylogeny of GCTs.

The primordial germ cells, first recognized in the normal embryonic yolk sac endoderm, migrate toward the gonadal folds. Germ cells that are topographically misplaced during migration normally do not survive and are probably eliminated by an immune mechanism.[26] If they survive and acquire neoplastic properties as a result of unknown oncogenic factors or mutant genes,[16] they may be designated as "neoplastic germ cells with totipotentiality." An analogous oncogenesis may occur within the gonadal folds, which are the normal destination of the migrating, primordial germ cells. Such neoplastic germ cells have been found and referred to as "intratubular malignant germ cells."[18]

According to Takei and Pearl,[26] germinomas (seminomas, dysgerminomas) are formed by neoplastic germ cells without further differentiation. The interspersed lymphocytes in germinomas are probably an immune response to the neoplastic germ cells and may play a role in preventing their further differentiation. The totipotent neoplastic germ cells may mature along either embryonal or extraembryonal lines. Embryonal differentiation results in teratomatous elements. Along an extraembryonal line, trophoblastic differentiation results in the formation of choriocarcinoma;[26] the other line of extraembryonal differentiation is toward yolk sac tumor (endodermal sinus tumor) and embryonal carcinoma. The major structural difference between the two is that embryonal carcinoma is predominantly composed of anaplastic endodermal cells, which are only one of multiple elements in yolk sac tumor.[26]

All of these theories seem to be based on a premise that GCTs, except for germinoma, arise by parthenogenetic fertilization of the germ cells.[30] Is it possible that such parthenogenesis really occurs in the human body?

According to some patholgists, this parthenogenesis may seem to be self-evident. Ashley[2] has suggested that there are two groups of teratomas. He writes, "One type seen in the gonads and possibly in the posterior abdominal wall is derived from germ cells by a process of parthenogenesis. The second type seen in the sacrococcygeal region, the head, and the chest is related to sequestration of cells of the blastula before differential blocking of the genome has occurred, and may be regarded as a derivative of an incomplete conjoined twin." However, the intracranial teratomas that my colleagues and I have examined, showed histological features similar to gonadal teratomas.

According to the vast statistical data accumulated by the Brain Tumor Registry of Japan,[6] an intracranial teratoma has two peaks in its age distribution: one peak is in the neonatal and infancy period (10.4\%) and the other peak is in the childhood period between age 5 to 14 years $(48.1 \%)$. It may be possible that teratoma in the first peak may be regarded as fetus-in-fetu and teratoma in the second peak derives from germ cells. This theory, however, has no proof. Arias-Bernal and Jones[1] studied chromosomes of a malignant ovarian teratoma. They commented, "the finding of chromosomes in the near diploid range again fails to support the idea that teratomas are possibly of germ cell origin in the ovocyte stage after chromosome reduction" and, "if teratomas arise from germ cells, it is likely that the 
origin in the ovary, at least, is from a cell in the oogonium stage, or even earlier. It does not seem necessary to invoke the concept of parthenogenesis or autofertilization to explain the finding of 46 chromosomes."

Another weakness of the germ cell theory is that there has been no explanation of the mechanism by which the primordial germ cells are incorporated into the cranial cavity, in view of the fact that the route of their physiological migration is so far apart from the cranial cavity.

\section{EXPERIMENTAL AND CLINICAL DATA FOR AND AGAINST THE GERM CELL THEORY}

Stevens[25] showed that $82 \%$ of 12 half-day genital ridges of strain $129 / \mathrm{Sv}-\mathrm{SI}{ }^{\mathrm{J}}$ male mice fetuses grafted to the testes of adult males of the same strain developed testicular teratomas. This is a much higher incidence than that which occurs spontaneously. This effect was not observed when the ridges were grafted to the spleen or in intratesticular grafts of 13 half-day genital ridges. It has been maintained that this experiment proved the totipotency of the primordial germ cells located in the genital ridge or, at least, derivation of teratoma from the primordial germ cells. It is, however, probable that the grafted genital ridge contained by far greater numbers of other primitive embryonic cells than the primordial germ cells and that those primitive embryonic cells gave rise to teratoma.

Kleinsmith and Pierce[14] observed in mice that single embryonal carcinoma cells were multipotential and capable of producing all the somatic tissues of a well-differentiated teratocarcinoma. Stevens'[24] experimental results revealed that embryoid bodies derived from the testicular teratomas of the $129 / \mathrm{Sv}$ SI ${ }^{\mathrm{J}}$ strain of mice had similarities in embryonic potency as well as in morphology to normal mouse embryo. Both of these findings support the aspect of germ cell theory maintaining that embryonal carcinoma may give rise to various types of tumors. Moreover, these findings cast favorable light on Askanazy's theory[3] that well-differentiated somatic tissues of teratomas develop by embryonic differentiation from either a single multipotential type of cell or from a group of cells composed of representatives of each of the embryonic germinal layers. These findings, however, do not prove the germ cell origin of embryonal carcinoma cells or of multipotential stem cells.

The existence of so-called mixed GCTs may seem to favor the germ cell theory. For instance, finding that a tumor composed of germinoma and teratoma may seem to warrant the assumption that the teratoma component has derived from germinoma. There is, however, no proof of this. Twenty-one percent (12 of 57) of mixed tumors in our series had no germinoma components[15] and 84\% (48 of 57) of those had no embryonal carcinoma components.

The fact that most contradicts the germ cell theory is the biological behavior of these GCTs. Clinically, these neoplasms are classified in relative order of increasing malignant behavior as follows:[4,12,15] germinoma, teratoma (including immature or malignant), embryonal carcinoma, yolk sac tumor (endodermal sinus tumor), and choriocarcinoma.

According to the germ cell theory, choriocarcinoma and yolk sac tumor (endodermal sinus tumor) should be the most differentiated among GCTs, and yet they are the most malignant. Germinoma, which should be the most undifferentiated, is actually a rather benign tumor. This contradicts the axiom of general oncology that the more undifferentiated a tumor is the more malignant it is and that the more differentiated a tumor is the more benign it is. In order to solve this contradiction, we propose working hypotheses in the subsequent section. 


\section{PERSONAL EXPERIENCES WITH INTRACRANIAL GCTS}

\section{Statistical Results}

Through 1994, we studied 153 cases of histologically verified intracranial GCTs.[15] We excluded from the study some of the two-cell pattern tumors in the pineal or suprasellar (neurohypophysis) region that we thought were tumors of pineal parenchyma origin (pineocytoma with lymphocytic infiltration) according to criteria stated elsewhere.[23] Ninety-six cases $(62.7 \%)$ displayed one type of histological composition in $98 \%$ or more of the examined sections, such as mature and immature teratoma, teratoma with malignant transformation, germinoma, embryonal carcinoma, yolk sac tumor (endodermal sinus tumor), and choriocarcinoma. Fifty-seven cases (37.3\%) were the so-called mixed GCTs (49 cases) and germinoma with syncytiotrophoblastic giant cells (STGCs) (eight cases). The mean patient age was 16.1 \pm 8.1 years. Seventy-eight tumors $(51.0 \%)$ were located in the pineal region, $46(30 \%)$ in the neurohypophysis (suprasellar) region, $16(10.5 \%)$ in other areas, and $13(8.5 \%)$ in multiple locations. There were 122 men $(79.7 \%)$ and 31 women $(20.3 \%$ ) (male/female ratio $4: 1$ ).

Mixed GCTs were composed of various combinations of two or more types of GCTs. Germinoma and teratoma components were most frequently seen. In the whole group of mixed GCTs, germinoma components were found in $79 \%$, teratoma components in $63 \%$, yolk sac tumor components in $33.3 \%$, and embryonal carcinoma components in $15.8 \%$.

Gender and tumor location distribution of GCTs were interesting. Among 78 tumors located in the pineal region, 76 were in men and only two were in women, whereas in the neurohypophysis (suprasellar) region 21 tumors were in men and 25 were in women. Therefore, there was a pronounced male predominance of GCTs in the pineal region and a slight female predominance of those in the neurohypophysis region.

It is well known that occipital encephalocele is predominant in women.[11] This may suggest that closure of the anterior neuropore occurs later in women than in men. If so, enfolded embryonic cells may reach deeper in the midline (neurohypophysis region) in women than in men, and those cells in males may reach a shallow part of the neural tube, that is, in the pineal region. This may explain the previously stated findings about GCTs, male predominance of these tumors in the pineal region, and slight female predominance of the tumors in the neurohypophysis region.

\section{Survival Rates of Patients With GCTs}

The survival rates (using the Kaplan-Meier method) in our series of patients with so-called intracranial GCTs treated by surgery, postoperative radiation therapy and/or chemotherapy were shown in our previous papers.[15,22] All patients with choriocarcinoma died within 2 years. Patients with yolk sac tumor (endodermal sinus tumor) and embryonal carcinoma also showed poor results. The 5- and 10-year survival rates for patients with mature teratoma were $92.9 \%$ each. Those with immature and malignant teratoma showed 5- and 10-year survival rates of 70.7\% each. Patients with germinoma showed a 5-year survival rate of $95.4 \%$ and a 10 -year survival rate of $92.7 \%$.

Analysis of these findings clearly indicated that patients with choriocarcinoma, embryonal carcinoma, and yolk sac tumor demonstrated the lowest survival rates and the tumors were therefore malignant, whereas patients with germinoma and mature teratoma had longer survival rates (10-year survival rates of nearly $93 \%$ each)[15] and their tumors were benign. Survival rates for patients with immature and malignant teratomas and germinoma with other components (such as STGCs) fell between the rates of 
the previously mentioned malignant and the benign groups. These data contradict the germ cell theory according to which choriocarcinoma and yolk sac tumor are most differentiated and should be most benign.

\section{PATHOGENETIC MECHANISMS OF GCTS}

\section{Hypothesis I. Nature of Tumors: Histogenetic Stages Compared With Ontogenetic Stages}

As is well known, in the Bailey-Cushing classification of gliomas or neuroectodermal tumors, those composed of cells resembling the cells in earlier stages of histogenesis are more malignant than those resembling the cells in later stages of histogenesis.

However, in so-called GCTs, we may assume the following based on our experiences: tumors composed of cells resembling the cells that appear in earlier stages of embryogenesis (ontogenesis) are more malignant than those composed of cells resembling the cells that appear in later stages of embryogenesis (ontogenesis).[22]

Each of the so-called GCTs may represent the neoplastic equivalent of an embryonic stage of development (Fig. 2): the trophoblast (choriocarcinoma) which appears as early as in the stage involving blastocyst formation, the yolk sac endoderm (yolk sac tumor or endodermal sinus tumor), the pluripotent stem cell of the embryo proper (embryonal carcinoma), the embryonic differentiated cell (teratoma), and the primordial germ cell (germinoma). With the exception of germinoma, these tumors may not originate from one single type of cell, namely, the primordial germ cell.

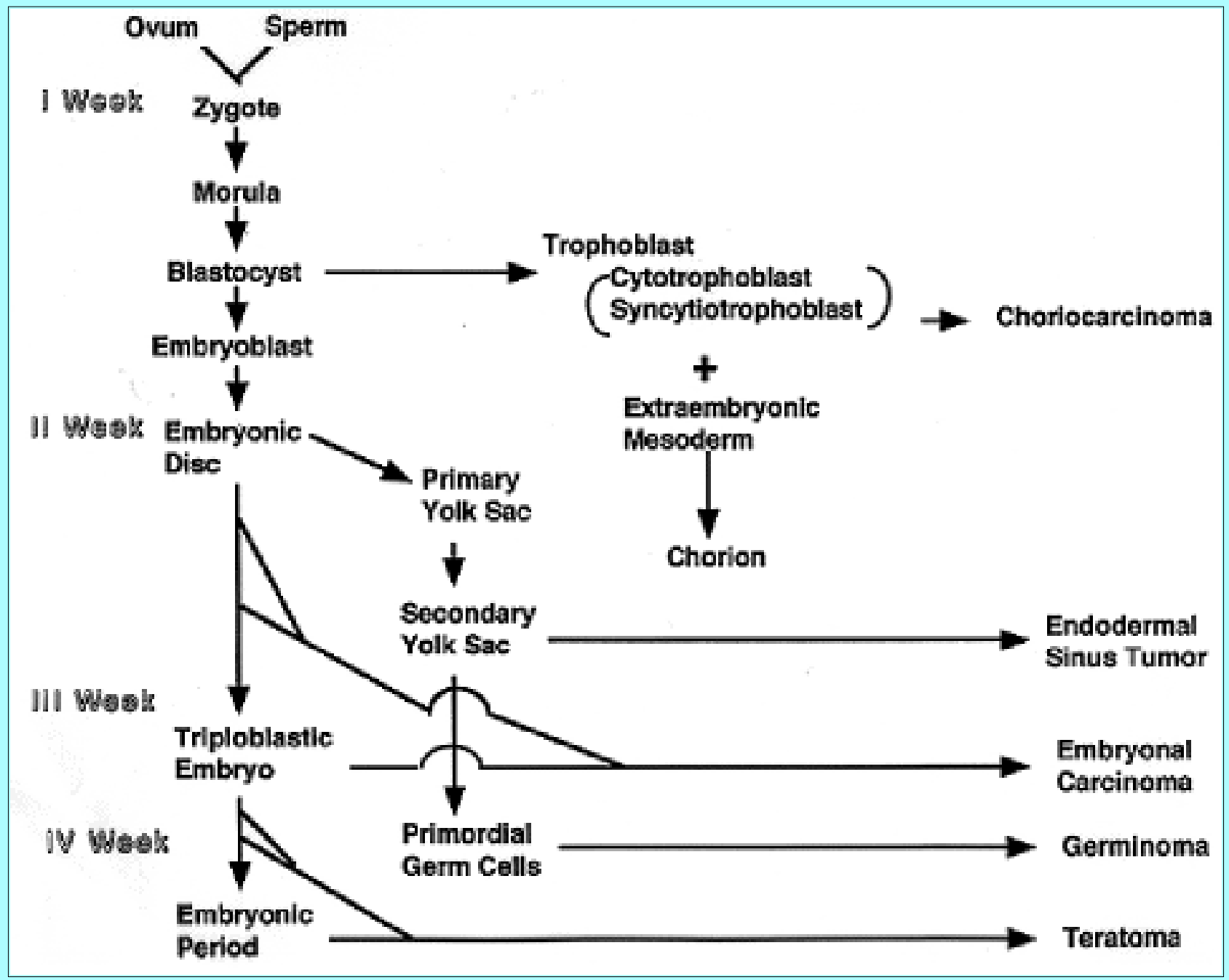


Fig. 2. Diagram depicting proposed hypothesis: embryonic development and possible neoplasms that may arise from the misinvolved-misenfolded cells. (Reprinted with permission of Sano K: So-called intracranial germ cell tumours: are they really of germ cell origin? Br J Neurosurg 9:391-401, 1995.)

Therefore, the true GCT may be only one: germinoma. The other so-called GCTs should be regarded as enfolded cell-derived dysembryogenetic tumors as stated later.

\section{Hypothesis II. Incorrect Involvement and Enfoldment}

In 1882, Julius Cohnheim,[5] propounded a theory of tumorigenesis in which neoplasms develop from the embryonic cell rests (embryonale anlage, or "verirrte Keime") that do not participate in the formation of normal surrounding tissues and are alien to the environment. This older theory may still be true with dysembryogenetic or malformative tumors. For instance, craniopharyngioma is believed, by many authors, to arise from the epithelial cell rests of Rathke's pouch; that is, this tumor is thought to be derived from the embryonic cell rests in situ. No such embryonic cell rests corresponding to each of the so-called GCTs normally exist in the cranial cavity.

Why these tumors arise in the cranial cavity may be explained by the "misinvolvement-misenfoldment" hypothesis.[23] At the end of the 1st week of embryogenesis, implantation of the blastocyst into the uterine wall commences and trophoblasts start proliferating rapidly. In the beginning of the 2nd week, the bilaminar embryonic disc is formed from the inner cell mass of the blastocyst.[17] The embryonic disc is closely attached to the cytotrophoblast, the syncytiotrophoblast, the amnion cells, and the yolk sac (primary and secondary) endoderm at its lateral margins so that cells of these structures may be misplaced in the disc edges.[22] In the beginning of the 3rd week of embryogenesis, the primitive streak appears at the caudal part of the embryonic disc, formed by the movement of proliferating embryonic disc cells toward the midline entering the primitive groove.[17] These cells leave the basal layer of the primitive groove, migrate laterally between the embryonic ectoderm and endoderm, and become organized into a layer called the intraembryonic mesoderm. Some misplaced cells of the embryonic disc, such as those of the adjacent yolk sac endoderm, the trophoblastic layer, or the amnion (produced by the cytotrophoblast), may be involved in this movement and migrate with the moving cells of the mesoderm, especially the lateral mesoderm to the future cranial area ("misinvolvement"). Around the 18th day, when the neural plate is formed and then starts folding to form the neural tube, these migrated cells may be enfolded into the neuraxis ("misenfoldment"), and from these cells the previously mentioned so-called GCTs may later develop.[22,23] The primordial germ cells first recognized as such by the end of the 3rd week (the 21st day) or the beginning of the 4th week (the 22nd day) in the yolk sac endoderm and near the origin of the allantois should be already present in the yolk sac endoderm before that date and may migrate with lateral mesoderm cells to the cranial area where they are misenfolded into the neuraxis and give rise to germinoma. It is also possibile that the primordial germ cells, during their physiological migration to the gonadal ridge, may be involved in a group of moving lateral mesoderm cells and may migrate to the cranial area to be enfolded into the neuraxis.[22,23] The existence of mixed GCTs of various combinations of cell components can also easily be understood. The recently described combination of germinoma and STGCs[29,31] can be interpreted as follows: the syncytiotrophoblastic cells and the primordial germ cells (yolk sac endoderm cells) are involved together in the lateral mesoderm stream moving to the future cranial area and are misenfolded into the cranial cavity. In these tumors, cytotrophoblasts are not present, whereas in choriocarcinoma or mixed tumors with choriocarcinomatous cells STGCs, which probably derive from cytotrophoblasts, are seen. However, 
there was no combination of germinoma and choriocarcinoma (cytotrophoblasts) in our series.[15] The existence of germinoma with STGCs without cytotrophoblastic cells may indicate that the coexistence of germinoma and syncytiotrophoblastic cells is only incidental (incidental misinvolvement). Most of these misinvolved cells are probably destroyed by immune mechanisms of the body, and only a small percentage of the cells reach the cranial cavity. Figure 2 again shows the relationship between each stage of the embryonic development and expectant neoplasms that will arise from misinvolved-misenfolded cells. This schema can explain the diversity of so-called GCTs and their nature (grades of malignancy). There is no reason to believe that all these tumors must be attributed to one single origin, the primordial germ cells, as shown in Fig. 1 which illustrates the twisted, improbable pathogenetic lines.

\section{References}

1. Arias-Bernal L, Jones HW Jr: Chromosomes of a malignant ovarian teratoma. Am J Obstet Gynecol 100:785-789, 1968

2. Ashley JB: Origin of teratomas. Cancer 32:390-394, 1973

3. Askanazy M: Die Teratome nach ihrem Bau, ihrem Verlauf, ihrer Genese und im Vergleich zum experimentellen Teratoid. Verhandl Dtsch Pathol Gesellsch 11:39-82, 1907

4. Brodeur GM, Howarth CB, Pratt CB, et al: Malignant germ cell tumors in 57 children and adolescents. Cancer 48:1890-1898, 1981

5. Cohnheim JF: Vorlesungen über allgemeine Pathologie. Berlin: Hirschwald, 1882, Vol 1, pp 723-796

6. Committee of Brain Tumor Registry of Japan: Age distribution of primary brain tumors. Neurol Med Chir 32:396-409, 1992

7. Dixon FJ, Moore RA: Testicular tumors. A clinicopathological study. Cancer 6:427-454, 1953

8. Friedman NB: The comparative morphogenesis of extragenital and gonadal teratoid tumors. Cancer 4:265-276, 1951

9. Friedman NB: Germinoma of the pineal. Its identity with germinoma ("seminoma") of the testis. Cancer Res 7:363-368, 1947

10. Friedman NB, Moore RA: Tumors of the testis. A report on 922 cases. Mil Surgeon 99:573-593, 1946

11. Guthkelch AN: Occipital cranium bifidum. Arch Dis Child 45:104-109, 1970

12. Jennings MT, Gelman R, Hochberg F: Intracranial germ-cell tumors: natural history and pathogenesis. J Neurosurg 63:155-167, 1985

13. Kleihues P, Burger PC, Scheithauer BW: The new WHO classification of brain tumours. Brain Pathol 3:255-268, 1993

14. Kleinsmith LJ, Pierce GB Jr: Multipotentiality of single embryonal carcinoma cells. Cancer Res 24:1544-1548, 1964 
15. Matsutani M, Sano K, Takakura K, et al: Primary intracranial germ cell tumors: a clinical analysis of 153 histologically verified cases. J Neurosurg 86:446-455, 1997

16. Mintz B, Fleischman RA: Teratocarcinomas and other neoplasms as developmental defects in gene expression. Adv Cancer Res 34:211-278, 1981

17. Moore KL, Persaud TVN: The Developing Human. Clinically Oriented Embryology, ed 5. Philadelphia: WB Saunders, 1993

18. Mostofi FK: Pathology of germ cell tumors of testis: a progress report. Cancer 45 (Suppl 7):1735-1754, 1980

19. Rubinstein LJ: Cytogenesis and differentiation of pineal neoplasms. Hum Pathol 12:441-448, 1981

20. Russell DS: The pinealoma: its relationship to teratoma. J Pathol Bacteriol 56:145-150, 1944

21. Sano K: Pineal region tumors: problems in pathology and treatment. Clin Neurosurg 30:59-91, 1983

22. Sano K: So-called intracranial germ cell tumours: are they really of germ cell origin? $\mathbf{B r} \mathbf{J}$

Neurosurg 9:391-401, 1995

23. Sano K, Matsutani M, Seto T: So-called intracranial germ cell tumours: personal experiences and a theory of their pathogenesis. Neurol Res 11:118-126, 1989

24. Stevens LC: Embryonic potency of embryoid bodies derived from a transplantable testicular teratoma of the mouse. Dev Biol 2:285-297, 1960

25. Stevens LC: Experimental production of testicular teratomas in mice. Proc Natl Acad Sci USA 52:654-661, 1964

26. Takei Y, Pearl GS: Ultrastructural study of intracranial yolk sac tumor: with special reference to the oncologic phylogeny of germ cell tumors. Cancer 48:2038-2046, 1981

27. Teilum G: Classification of endodermal sinus tumor (mesoblastoma vitellinum) and so-called 'embryonal carcinoma' of the ovary. Acta Pathol Microbiol Scand 64:407-429, 1965

28. Teilum G: Endodermal sinus tumor of the ovary and testis -comparative morphogenesis of the so-called mesonephroma ovarii (Schiller) and extraembryonic (yolk sac-allantoic) structure of the rat's placenta. Cancer 12:1092-1105, 1959

29. Ueda G, Hamanaka N, Hayakawa K, et al: Clinical, histochemical and biochemical studies of an ovarian dysgerminoma with trophoblasts and Leydig cells. Am J Obstet Gynecol 114:748-754, 1972

30. Woodruff JD: Embryology and its relation to tumorigenesis in the ovary and adjacent structures, in Mack HC (ed): The Ovary. Springfield, Ill: Charles C Thomas, 1968, pp 82-105

31. Zaloudek CJ, Tavassoli FA, Norris HJ: Dysgerminoma with syncytiotrophoblastic giant cells. A histologically and clinically distinctive subtype of dysgerminoma. Am J Surg Pathol 5:361-367, 1981

32. Zülch KJ: Brain Tumors: Their Biology and Pathology, ed 3. Berlin: Springer-Verlag, 1986, pp 414-424 
Manuscript received May 18, 1998.

Accepted in final form June 24, 1998.

Address reprint requests to: Keiji Sano, M.D., Department of Neurosurgery, Teikyo University School of Medicine, 2-11-1 Kaga, Itabashi-ku, Tokyo 173-8605, Japan.

\section{Table of Contents Return to N://0C@}

